06.3

\title{
Быстрые и медленные электромагнитные волны в продольно намагниченном тонкопленочном ферромагнитном метаматериале
}

\author{
() М.Д. Амельченко, С.В. Гришин Ф, Ю.П. Шараевский \\ Саратовский национальный исследовательский государственный университет им. Н.Г. Чернышевского, Саратов, Россия \\ ฯ E-mail: sergrsh@yandex.ru
}

Поступило в Редакцию 8 апреля 2019г.

В окончательной редакции 8 апреля 2019г.

Принято к публикации 26 августа 2019г.

\begin{abstract}
Приводятся результаты теоретического исследования электродинамических характеристик быстрых и медленных электромагнитных волн (ЭМВ), распространяющихся в метаматериале, состоящем из ферромагнитной пленки, внутри которой располагается периодическая решетка из тонких металлических проволочек. Установлено, что ферромагнитный тонкопленочный метаматериал обладает свойствами левой среды на частотах медленной ЭМВ. Показано, что в ферромагнитном метаматериале по сравнению с обычной ферромагнитной пленкой частоты отсечки быстрых и медленной ЭМВ сдвигаются в более высокочастотный диапазон, а сами ЭМВ становятся сильно замедленными.
\end{abstract}

Ключевые слова: метаматериалы, левая среда, обратная волна, ферромагнетик, спиновые волны.

DOI: 10.21883/PJTF.2019.23.48712.17830

Известно, что метаматериалы представляют собой искусственно созданные среды со свойствами, не встречающимися у обычных природных сред [1-3]. Среди метаматериалов особый класс составляют так называемые „левые с среды, у которых диэлектрическая $\varepsilon$ и магнитная $\mu$ проницаемости одновременно имеют отрицательные значения [3]. В левой среде векторы напряженности электрического и магнитного полей совместно с волновым вектором составляют левую тройку векторов. Это приводит к распространению в такой среде обратной электромагнитной волны (ЭМВ), векторы фазовой и групповой скоростей которой направлены в противоположные стороны. В микроволновом диапазоне для создания метаматериалов в виде левых сред обычно используются периодические структуры из тонких металлических проволочек и разрезных кольцевых резонаторов, период следования которых намного меньше длины ЭМВ [3,4]. Периодическая система из тонких металлических проволочек позволяет создать структуру плазмонного типа, эффективная диэлектрическая проницаемость которой $\varepsilon_{e f f}$ может быть как положительной, так и отрицательной величиной. Периодическая система из разрезных кольцевых резонаторов традиционно используется для создания искусственных магнитных сред с отрицательным значением эффективной магнитной проницаемости. Однако помимо искусственно созданных магнитных сред в природе существуют и естественные магнитные среды в виде ферромагнетиков, антиферромагнетиков и ферримагнетиков (или ферритов), у которых при наложении внешнего постоянного магнитного поля $H_{0}$ магнитная проницаемость $\mu$ становится отрицательной $[5,6]$. Частотная область, в которой $\mu<0$, приходится на диапазон частот существования правополяризованных медленных ЭМВ, которые являются волнами намагниченности; они получили название магнитостатических волн. В случае продольного намагничивания, когда векторы внешнего постоянного магнитного поля и фазового фронта ЭМВ являются коллинеарными, в ферромагнетике распространяются медленные обратные объемные ЭМВ [6]. Однако указанные волны существуют в диапазоне частот, в котором $\mu<0$, а $\varepsilon>0$. $\mathrm{B}$ настоящей работе показана возможность создания из ферромагнитной среды ферромагнитного метаматериала со свойствами левой среды $(\mu<0$ и $\varepsilon<0)$, в которой распространяются сильно замедленные обратные объемные ЭМВ.

На рис. 1, а приведено схематическое изображение анализируемого ферромагнитного метаматериала. Он представляет собой продольно намагниченный ферромагнитный слой, в котором в качестве периодической структуры используется система из тонких металлических проволочек с периодом следования $T$. Предполагается, что период следования структуры намного меньше длины ЭМВ, т. е. $T \ll \lambda$. В этом случае для описания диэлектрических свойств ферромагнетика в отсутствие потерь можно ввести эффективную диэлектрическую проницаемость среды с частотной зависимостью плазмонного типа [7]:

$$
\varepsilon_{e f f}(\omega)=1-\frac{\omega_{p}^{2}}{\omega^{2}},
$$

где $\omega_{p}=\sqrt{2 \pi} c /[T \sqrt{\ln (T / r)}]$ - плазменная частота, $c$ - скорость света в вакууме, $r$ - радиус проволоки, $\omega=2 \pi f-$ круговая частота, $f-$ линейная частота. Из выражения (1) следует, что $\varepsilon_{e f f}>0$ при $\omega>\omega_{p}$ и $\varepsilon_{\text {eff }}<0$ при $\omega<\omega_{p}$.

Для расчета дисперсионных характеристик (ДХ) ЭМВ, распространяющихся в продольно намагниченном 

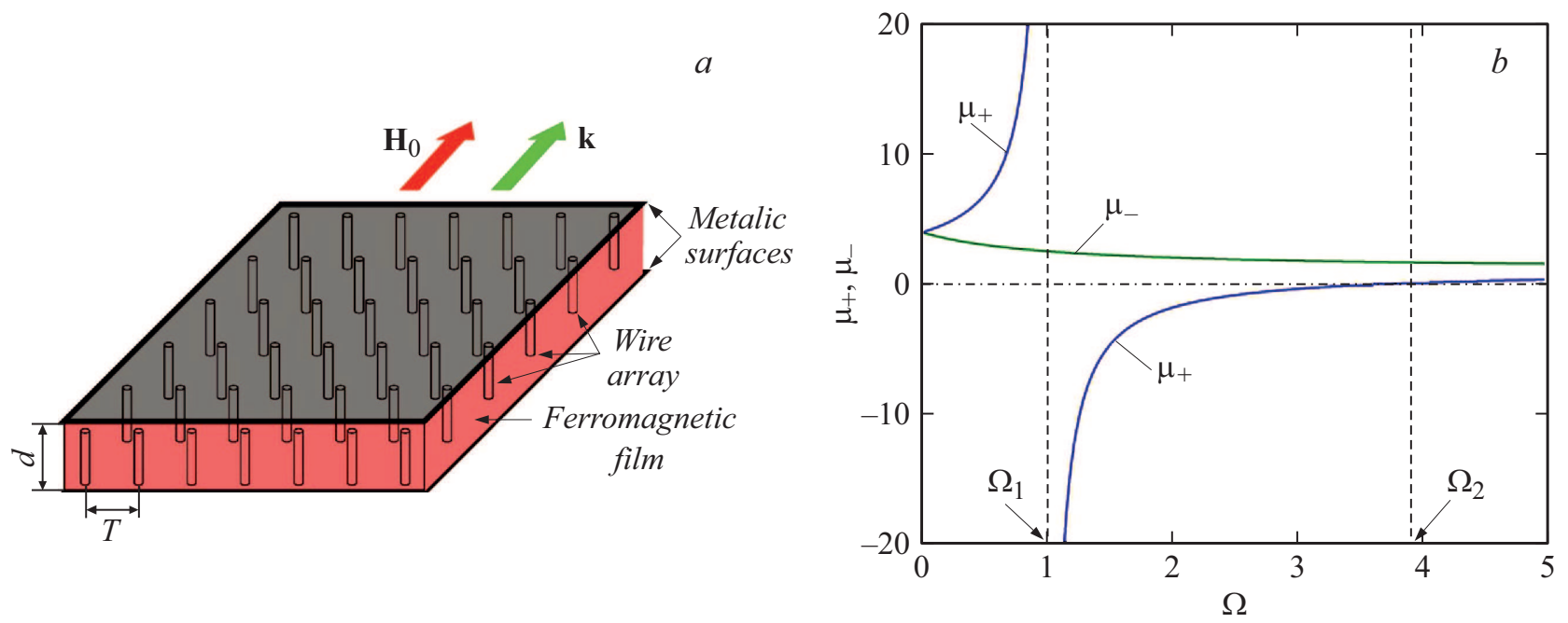

Рис. 1. $a-$ схематическое изображение ферромагнитного метаматериала; $b-$ зависимость правополяризованной компоненты $\mu_{+}$ и левополяризованной компоненты $\mu_{-}$тензора магнитной проницаемости от безразмерной частоты $\Omega=\omega / \omega_{H}$. Расчеты выполнены для $H_{0}=47.76 \mathrm{kA} / \mathrm{m}$ и $4 \pi M_{0}=0.175 \mathrm{~T}$.

ферромагнитном метаматериале, воспользуемся известным дисперсионным уравнением для продольно намагниченного ферромагнетика $[8,9]$. Данное уравнение с учетом эффективной диэлектрической проницаемости среды примет следующий вид:

$$
\begin{aligned}
& -\mu k_{z}^{4}-\left[(1+\mu) k^{2}-\left(\mu^{2}+\mu-\mu_{a}^{2}\right) k_{0}^{2} \varepsilon_{e f f}\right] k_{z}^{2} \\
& =k^{4}-2 \mu k^{2} k_{0}^{2} \varepsilon_{e f f}+\left(\mu^{2}-\mu_{a}^{2}\right) k_{0}^{4} \varepsilon_{e f f}^{2},
\end{aligned}
$$

где $\mu=\left[\omega_{H}\left(\omega_{H}+\omega_{M}\right)-\omega^{2}\right] /\left(\omega_{H}^{2}-\omega^{2}\right)-$ диагональная компонента тензора магнитной проницаемости, а $\mu_{a}=\omega_{M} \omega /\left(\omega_{H}^{2}-\omega^{2}\right)-$ его недиагональная компонента, $\omega_{H}=\gamma H_{0}-$ частота ферромагнитного резонанса, $\omega_{M}=4 \pi \gamma M_{0}, \gamma$ - гиромагнитное отношение, $4 \pi M_{0}-$ намагниченность насыщения материала, $k_{0}=\omega / c-$ волновое число ЭМВ в вакууме, $k$ - продольное волновое число ЭМВ в ферромагнитном метаматериале, $k_{z}-$ поперечное волновое число, вид которого находится из граничных условий.

Будем рассматривать ферромагнитный метаматериал, граничащий с обеих сторон с идеально проводящими металлическими экранами. Для продольно намагниченного полоскового волновода, полностью заполненного ферромагнетиком, такая задача была решена строго в [9]. В частности, в [9] было показано, что при продольном намагничивании в металлизированном с обеих сторон ферромагнитном слое могут распространяться как квази-ТЕ-, так и квази-ТМ-волны, обладающие разными значениями поперечного волнового числа. В то же время при решении уравнений Максвелла в магнитостатическом приближении, которое является справедливым для тонких ферромагнитных пленок, была получена функция поперечного волнового числа для квази-ТЕ-волны в виде [6]:

$$
k_{z}=n \pi / d,
$$

где $d$ - толщина тонкопленочного ферромагнетика, $n-$ номер моды объемной ЭМВ.

Дисперсионное уравнение (2) с учетом (1) приводится к полиномиальному виду относительно частоты $\omega$. Полученный полином представляет собой бикубическое уравнение, которое имеет следующий вид:

$$
A_{1} \omega^{6}+A_{2} \omega^{4}+A_{3} \omega^{2}+A_{4}=0
$$

где

$$
\begin{gathered}
A_{1}=1, \\
A_{2}=-\left\{2\left[c^{2}\left(k^{2}+k_{z}^{2}\right)+\omega_{p}^{2}\right]+\left(\omega_{H}+\omega_{M}\right)^{2}\right\}, \\
A_{3}=c^{2}\left(k^{2}+k_{z}^{2}\right)\left[c^{2}\left(k^{2}+k_{z}^{2}\right)+2 \omega_{p}^{2}\right]+\omega_{p}^{4}+\left(\omega_{H}+\omega_{M}\right) \\
\times\left[\left(c^{2} k_{z}^{2}+2 \omega_{p}^{2}\right)\left(\omega_{H}+\omega_{M}\right)+c^{2} \omega_{H}\left(2 k^{2}+k_{z}^{2}\right)\right], \\
A_{4}=-\left\langle\omega _ { p } ^ { 2 } ( \omega _ { H } + \omega _ { M } ) \left\{ c^{2}\left[2 k^{2} \omega_{H}+k_{z}^{2}\left(2 \omega_{H}+\omega_{M}\right)\right]\right.\right. \\
\left.+\omega_{p}^{2}\left(\omega_{H}+\omega_{M}\right)\right\}+\omega_{H} c^{4}\left(k^{2}+k_{z}^{2}\right) \\
\left.\times\left[k^{2} \omega_{H}+k_{z}^{2}\left(\omega_{H}+\omega_{M}\right)\right]\right\rangle .
\end{gathered}
$$

Уравнение (4) имеет три действительных решения, два из которых соответствуют так называемым быстрым объемным ЭМВ, фазовая скорость которых больше скорости света в свободном пространстве $\left(v_{p h}>c\right)$, а третье решение соответствует медленной объемной ЭМВ, фазовая скорость которой значительно меньше скорости света в свободном пространстве $\left(v_{p h} \ll c\right)$.

Известно [6], что одна из двух быстрых ЭМВ является левополяризованной волной, а другая быстрая ЭМВ и медленная ЭМВ являются правополяризованными волнами. Для право- и левополяризованных ЭМВ вводятся циркулярные компоненты тензора высокочастотной 

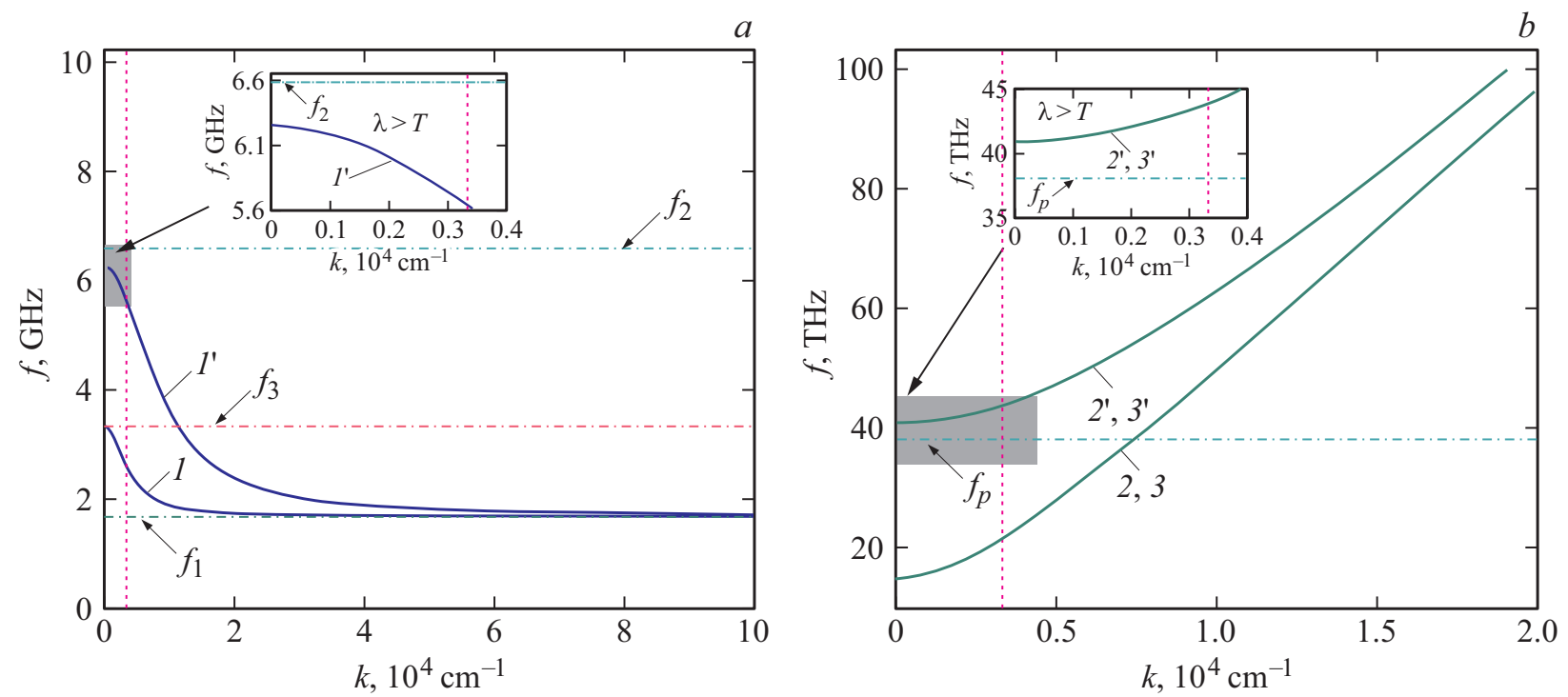

Рис. 2. Дисперсионные характеристики медленных $(a)$ и быстрых $(b)$ ЭМВ, распространяющихся в металлизированной ферромагнитной пленке (кривые $1-3)$ и в металлизированной пленке из ферромагнитного метаматериала (кривые $\left.1^{\prime}-3^{\prime}\right)$. Для расчета использовались следующие значения параметров системы: $d=10 \mu \mathrm{m}, H_{0}=47.76 \mathrm{kA} / \mathrm{m}, 4 \pi M_{0}=0.175 \mathrm{~T}, r=1 \mu \mathrm{m}$, $T=3 \mu \mathrm{m}$ и $n=1$. Пунктирной линией отмечено значение волнового числа, при котором $\lambda=T$. Штрихпунктирными линиями показаны граничные частоты $f_{1}, f_{2}, f_{3}$ спектра медленной ЭМВ и плазменная частота $f_{p}$, ограничивающая спектр быстрых ЭМВ в ферромагнитном метаматериале.

магнитной проницаемости, которые имеют следующий вид $[6]$ :

$$
\begin{aligned}
& \mu_{+}=1+\frac{\omega_{M}}{\omega_{H}-\omega}, \\
& \mu_{-}=1+\frac{\omega_{M}}{\omega_{H}+\omega},
\end{aligned}
$$

где $\mu_{+}-$правополяризованная компонента тензора $\overleftrightarrow{\mu}$ а $\mu_{-}$- его левополяризованная компонента.

На рис. 1, $b$ приведены частотные зависимости правои левополяризованной компонент магнитной проницаемости. Из анализа результатов, представленных на рис. $1, b$, следует, что правая циркулярная составляющая $\mu_{+}$магнитной проницаемости, связанная с правополяризованным высокочастотным магнитным полем, проходит через резонанс при $\Omega=\Omega_{1}=1$. В то же время левая циркулярная составляющая $\mu_{-}$через резонанс не проходит и монотонно изменяется с изменением частоты $\omega$ или магнитного поля $H_{0}$. Кроме того, правая циркулярная составляющая проницаемости $\mu_{+}$меняет свой знак не только на частоте $\Omega_{1}$, где $\mu_{+} \rightarrow \pm \infty$, но и на другой характерной частоте $\Omega_{2}=1+\Omega_{M}$ (где $\left.\Omega_{M}=\omega_{M} / \omega_{H}\right)$, на которой $\mu_{+}=0$. Таким образом, высокочастотная магнитная проницаемость для быстрой левополяризованной ЭМВ всегда больше нуля $\left(\mu_{-}>0\right)$ во всем диапазоне частот, в то время как высокочастотная магнитная проницаемость для правополяризованных ЭМВ больше нуля $\left(\mu_{+}>0\right)$ на частотах $\Omega<1$ и $\Omega>\Omega_{2}$, на которых распространяются только быстрые ЭМВ. В интервале частот $\Omega_{1}<\Omega<\Omega_{2}$, где существуют медленные ЭМВ, высокочастотная магнитная проницаемость меньше нуля $\left(\mu_{+}<0\right)$.
На рис. 2, $a$ приведены результаты расчета ДХ быстрых и медленных объемных ЭМВ, полученных на основе решения дисперсионного уравнения (4) с учетом (3). Из представленных на рис. 2, $a$ результатов расчета следует, что в обычном ферромагнетике $\left(f_{p}=0\right)$ ДХ медленной объемной ЭМВ существует в интервале частот $f_{1}=f_{H}<f \leqslant f_{3}=\left[f_{H}\left(f_{H}+f_{M}\right)\right]^{1 / 2}$. В случае ферромагнитного метаматериала, когда значение плазменной частоты больше значения верхней границы области частот, в которой $\mu_{+}<0\left(f_{p} \geqslant f_{2}=f_{H}+f_{M}\right)$, эффективная диэлектрическая проницаемость метаматериала становится отрицательной величиной $\left(\varepsilon_{e f f}<0\right)$ во всем интервале частот $f_{1}<f<f_{2}$. Это приводит к формированию на указанных частотах медленной обратной ЭМВ, частота отсечки которой $f_{0}$ при $k=0$ зависит от геометрии периодической структуры и при $T \rightarrow 0 \quad f_{0} \rightarrow f_{2}$. Таким образом, как следует из результатов, представленных на рис. $2, a$, диапазон существования медленной объемной ЭМВ в ферромагнитном метаматериале расширяется и занимает практически весь интервал частот $f_{1}<f<f_{2}$, в котором ферромагнитный метаматериал является левой средой. Однако условие $T \ll \lambda$ для медленной ЭМВ выполняется только в полосе частот $\sim 600 \mathrm{MHz}$. Что касается быстрых право- и левополяризованных ЭМВ, то, как следует из результатов, представленных на рис. 2, $b$, их частоты отсечки находятся в терагерцевой области, определяются плазменной частотой и отстроены относительно друг друга на незначительную величину $\sim 2 \mathrm{MHz}$. При этом обе частоты отсечки находятся выше частот отсечки быстрых ЭМВ, распространяющихся в 

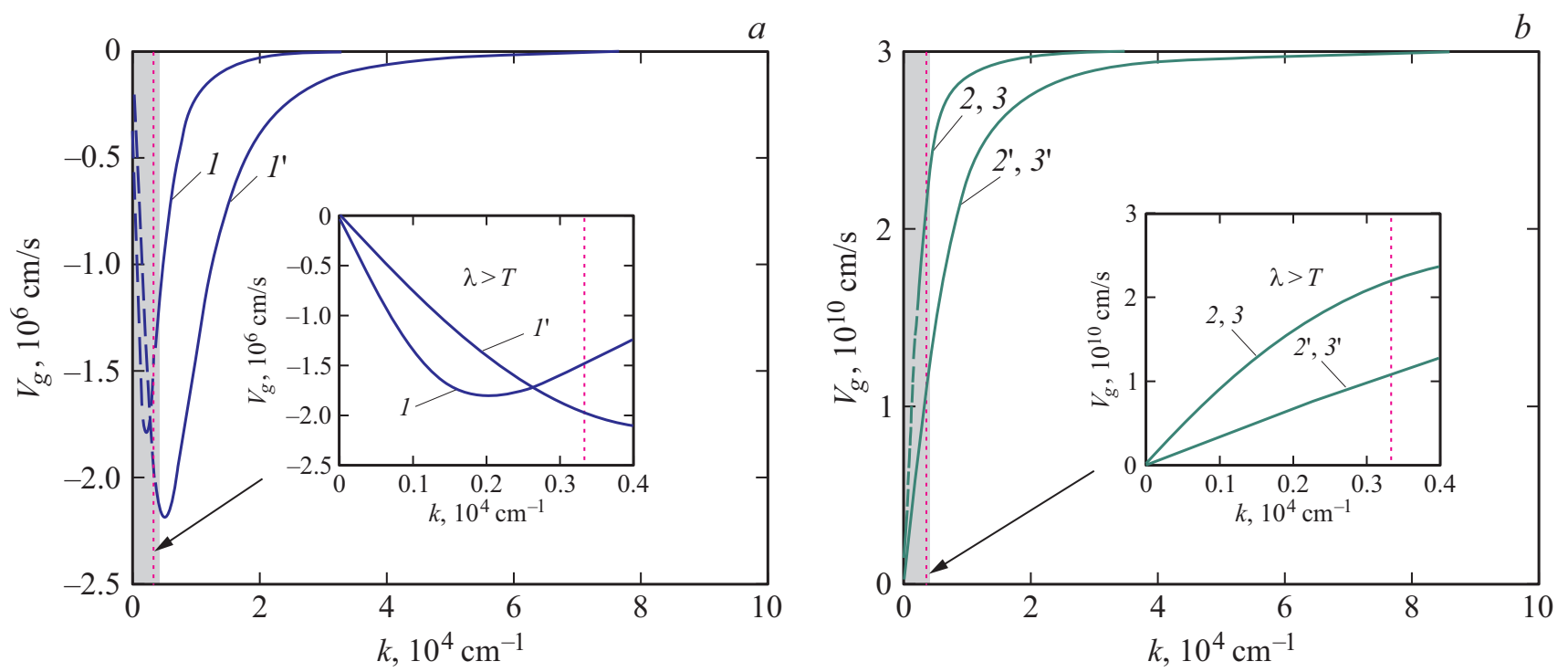

Рис. 3. Зависимости групповых скоростей от волнового числа медленных $(a)$ и быстрых $(b)$ ЭМВ, распространяющихся в металлизированной ферромагнитной пленке $(1-3)$ и в металлизированной пленке из ферромагнитного метаматериала (кривые $\left.1^{\prime}-3^{\prime}\right)$. Для расчета использовались следующие значения параметров системы: $d=10 \mu \mathrm{m}, H_{0}=47.76 \mathrm{kA} / \mathrm{m}$, $4 \pi M_{0}=0.175 \mathrm{~T}, r=1 \mu \mathrm{m}, T=3 \mu \mathrm{m}$ и $n=1$. Пунктирной линией отмечено значение волнового числа, при котором $\lambda=T$.

обычной ферромагнитной пленке. Условие $T \ll \lambda$ для быстрых ЭМВ выполняется в полосе частот $\sim 3 \mathrm{THz}$. На вставках к обоим рисункам приведены увеличенные фрагменты ДХ медленной и быстрых ЭМВ в области частот отсечки. Видно, что при $T \ll \lambda$ медленная ЭМВ, распространяющаяся на частотах, где $\mu<0$ и $\varepsilon<0$, имеет аномальную отрицательную дисперсию, а обе быстрые ЭМВ, распространяющиеся на частотах, где $\mu>0$ и $\varepsilon>0$, характеризуются положительной нормальной дисперсией.

На рис. 3 показаны зависимости групповых скоростей быстрых и медленных ЭМВ от волнового числа, рассчитанные для обычной ферромагнитной пленки и ферромагнитного метаматериала. Из сравнения результатов расчета следует, что как в случае медленных (рис. 3,a), так и в случае быстрых (рис. $3, b$ ) ЭМВ, распространяющихся в ферромагнитном метаматериале, значения их групповых скоростей становятся меньше значений групповых скоростей ЭМВ, распространяющихся в ферромагнитной пленке, практически во всей области волновых чисел, для которых выполняется условие $T \ll \lambda$. Таким образом, быстрые и медленные ЭМВ, распространяющиеся в тонкопленочном ферромагнитном метаматериале, становятся более замедленными по сравнению с ЭМВ, распространяющимися в обычной ферромагнитной пленке.

В заключение отметим, что проведенные в работе расчеты электродинамических характеристик быстрых и медленных ЭМВ дают возможность оценить перспективы использования ферромагнитных пленок для создания из них метаматериалов. В частности, при проведении численных исследований установлено, что спектр объем- ных быстрых и медленных ЭМВ, распространяющихся в ферромагнитном метаматериале, сдвигается вверх по частоте. При этом сами ЭМВ становятся более замедленными. Показано также, что в микроволновом диапазоне частот ферромагнитный метаматериал обладает свойствами левой среды, в которой распространяется обратная ЭМВ, причем диапазон частот, в котором ферромагнитный метаматериал является левой средой, зависит от величины внешнего постоянного магнитного поля и может перестраиваться по частоте.

\section{Финансирование работы}

Работа выполнена за счет гранта Российского научного фонда (проект № 19-79-20121).

\section{Конфликт интересов}

Авторы заявляют, что у них нет конфликта интересов.

\section{Список литературы}

[1] Веселаго В.Г. // УФН. 1967. Т. 92. № 7. С. 517-526.

[2] High A.A., Devlin R.C., Dibos A., Polking M., Wild D.S., Perczel J., de Leon N.P., Lukin M.D., Park H. // Nature. 2015. V. 522. N 7555. P. 192-196.

[3] Tong X.C. Functional metamaterials and metadevices. Springer Ser. in Materials Science. 2018. V. 262. 277 p.

[4] Вендик И.Б., Вендик О.Г. // ЖТФ. 2013. Т. 83. В. 1. С. 3-28.

[5] Вашковский А.В., Локк Э.Г. // УФН. 2004. Т. 174. № 6. C. 657-662. 
[6] Вашковский А.В., Стальмахов В.С., Шараевский Ю.П. Магнитостатические волны в электронике сверхвысоких частот. Саратов: Изд-во СГУ, 1993. $311 \mathrm{c.}$

[7] Pendry J.B., Holden A.J., Stewart W.J., Youngs I. // Phys. Rev. Lett. 1996. V. 76. N 25. P. 4773-4776.

[8] Гришин С.В., Шараевский Ю.П. // ЖТФ. 2001. Т. 71. В. 4. C. $95-98$.

[9] Микаэлян А.Л. Теория и применение ферритов на сверхвысоких частотах. М.-Л.: Госэнергоиздат, 1963. $664 \mathrm{c}$. 